\title{
MISE EN ÉVIDENCE DE L'ÉCOTYPE TRUITE DE MER DANS LES CAPTURES DE GRANDES TRUITES (SALMO TRUTTA L.) DU RHIN SUPÉRIEUR
}

\author{
P. ROCHE
}

Conseil Supérieur de la Pêche - 18, rue de Nomeny - 57158 MONTIGNY- LES-METZ

\section{RÉSUMÉ}

En 1989 et 1990, des écailles de 26 grandes truites ont été prélevées par des pêcheurs aux lignes dans le Rhin entre Iffezheim et Strasbourg, et dans l'Ill près de sa confluence avec le Rhin. La structure des écailles ainsi que leur teneur en strontium ont été comparées à celles d'écailles de truites sédentaires du Rhin.

22 truites avaient une structure d'écailles du type truite de mer et quatre truites s'apparentaient au type sédentaire. La teneur moyenne en strontium du groupe "mer" était de $394 \mu \mathrm{g} / \mathrm{g}$, valeur significativement plus élevée que celle du groupe "sédentaire" $(201 \mu \mathrm{g} / \mathrm{g})$. Un seul individu du type mer avait une valeur de strontium recoupant les valeurs du type sédentaire. La méthode de discrimination par l'analyse chimique des écailles est discutée, et des éléments sur l'origine et les caractéristiques des truites de mer du Rhin sont donnés.

\section{CHARACTERIZATION OF SEA TROUT AMONG CATCHES OF LARGE TROUT (SALMO TRUTTA L.) FROM THE RHINE}

\begin{abstract}
In 1989 and 1990, scale samples were collected from 26 large trout by anglers in the Rhine, between Iffezheim and Strasbourg, and in the Ill near its confluence to the Rhine. The structure and strontium content at these scales were compared with those of sedentary trout from the Rhine.

22 trout were found to have a scale structure of the sea trout type whereas four had scales of the sedentary type. The mean strontium content of the "sea" group was $394 \mu \mathrm{g} / \mathrm{g}$, significantly higher than the mean strontium content of the "sedentary" group $(201 \mu \mathrm{g} / \mathrm{g})$. A single fish from the sea type had a strontium content which was characteristic of the sedentary type. The distinction method using chemical analysis of the scales is discussed. Characteristics of the anadromous trout are given and the origin of this population is considered.
\end{abstract}

\section{INTRODUCTION}

La truite commune est présente en Europe sous trois formes : truite de rivière (généralement appelée truite fario), truite de lac et truite de mer. II s'agit vraisemblablement d'une seule espèce (Salmo trutta L.) possédant une grande capacité d'adaptation à différents milieux (BAGLINIĖRE, 1991). La truite de mer et la truite de lac, pélagiques au stade adulte, ont dans ces milieux une robe argentée marquée de croix noires. Leur robe, en particulier celle des mâles, se colore à nouveau lorsque ces poissons remontent en rivière pour se reproduire. La 
truite de rivière conserve généralement une robe colorée, mais elle peut devenir plus ou moins argentée dans les grands cours d'eau.

La présence de truites de mer dans le Rhin est signalée depuis la fin des années 1970 en Hollande (CAZEMIER, 1988 et 1991 ; GROOT, 1989) et en Allemagne (OLBRICH, 1984 ; WEIBEL, 1991 ; STEINBERG et LUBIENIECKI, 1991), tandis que les mêmes observations étaient faites dans la Meuse en Belgique, cours d'eau qui partage le même estuaire que le Rhin (PHILIPPART, 1983). De même en France, de grandes truites semblables à l'écotype marin sont capturées par pêche à la ligne ou au filet depuis 1975 dans le Rhin et dans l'Ill près de Strasbourg, à plus de $700 \mathrm{~km}$ de la mer (données non publiées). Bien que l'aspect de la robe et l'étude de la structure des écailles de ces individus confirment souvent leur appartenance au type truite de mer, il était nécessaire de recourir à une autre méthode pour attester leur éventuel séjour marin. L'analyse de la teneur en strontium des écailles permet généralement de distinguer les deux écotypes, le niveau de strontium étant plus élevé chez la forme marine que chez la forme dulçaquicole (BAGENAL et al., 1973; SALIOU, 1990). De plus cette méthode de discrimination semble réellement performante lorsque les individus marins ont séjourné plus d'un an en mer et sont remontés en rivière tôt dans la saison (MAISSE et BAGLINIËRE, 1990). L'analyse de la teneur en strontium des écailles a donc été réalisée sur un échantillon de grandes truites du Rhin. Les résultats sont présentés ainsi que les caractéristiques biométriques et scalimétriques de ces poissons.

\section{MATÉRIEL ET MÉTHODES}

En 1989 et 1990, 26 échantillons d'écailles de truites ont été prélevés par des pêcheurs à la ligne dans le Rhin depuis l'aval du barrage d'Iffezheim (premier obstacle depuis l'estuaire) jusqu'au barrage de Strasbourg, et dans l'ill au niveau de sa confluence avec le Rhin. Ce secteur d'une longueur de $50 \mathrm{~km}$ est situé à environ $700 \mathrm{~km}$ de la mer (Fig. 1). Des échantillons d'écailles ont également été prélevés sur trois truites capturées lors de pêches de sondage dans le Vieux-Rhin, dans un secteur situé à $80 \mathrm{~km}$ en amont de Strasbourg et séparé du premier par 7 grands barrages. Ces échantillons étaient destinés à fournir des valeurs-témoins de teneurs en strontium des truites sédentaires du Rhin.

La longueur totale des poissons a été mesurée. Le dosage du strontium a été réalisé sur environ $5 \mathrm{mg}$ d'écailles par spectrophotométrie d'absorption atomique selon la technique décrite par BAGENAL et al. (1973). L'interprétation des écailles de truites de mer a été faite et l'âge de rivière et de mer noté selon les critères proposés par RICHARD et BAGLINIĖRE (1990).

\section{RÉSULTATS}

- La lecture des écailles a permis de classer les truites en deux types selon le profil de croissance (Tableaux I et II) : 22 truites ont été classées dans le type "mer" (passage net en milieu à croissance rapide) et 4 truites dans le type "sédentaire" (croissance plus lente et régulière). Les 3 truites sédentaires capturées dans le Vieux-Rhin sont incluses au Tableau II afin de comparer leur teneur en strontium avec celles du type "sédentaire" capturées en aval de Strasbourg.

Les teneurs moyennes en strontium sont significativement différentes au seuil de $1 \%$ entre le type "mer" (394 $\mu \mathrm{g} / \mathrm{g}, \sigma=163)$ et le type "sédentaire" $(201 \mu \mathrm{g} / \mathrm{g}, \sigma=41)$. La différence entre les sous-échantillons "sédentaires aval" et "sédentaires Vieux-Rhin" n'est pas significative au seuil de $5 \%$. Seul un individu classé d'après la structure des écailles dans le type "mer" a une valeur de strontium $(131 \mu \mathrm{g} / \mathrm{g})$ recoupant celles du type "sédentaire" (Fig. 2). L'analyse du strontium est donc particulièrement cohérente avec l'analyse scalimétrique.

- La taille moyenne des individus de l'échantillon du type "mer" est de $631 \mathrm{~mm}$ $(\sigma=155)$ et elle est de $427 \mathrm{~mm}(\sigma=53)$ pour les quatre truites du type "sédentaire" capturées par les pêcheurs. Cette différence est significative au seuil de $5 \%$. 


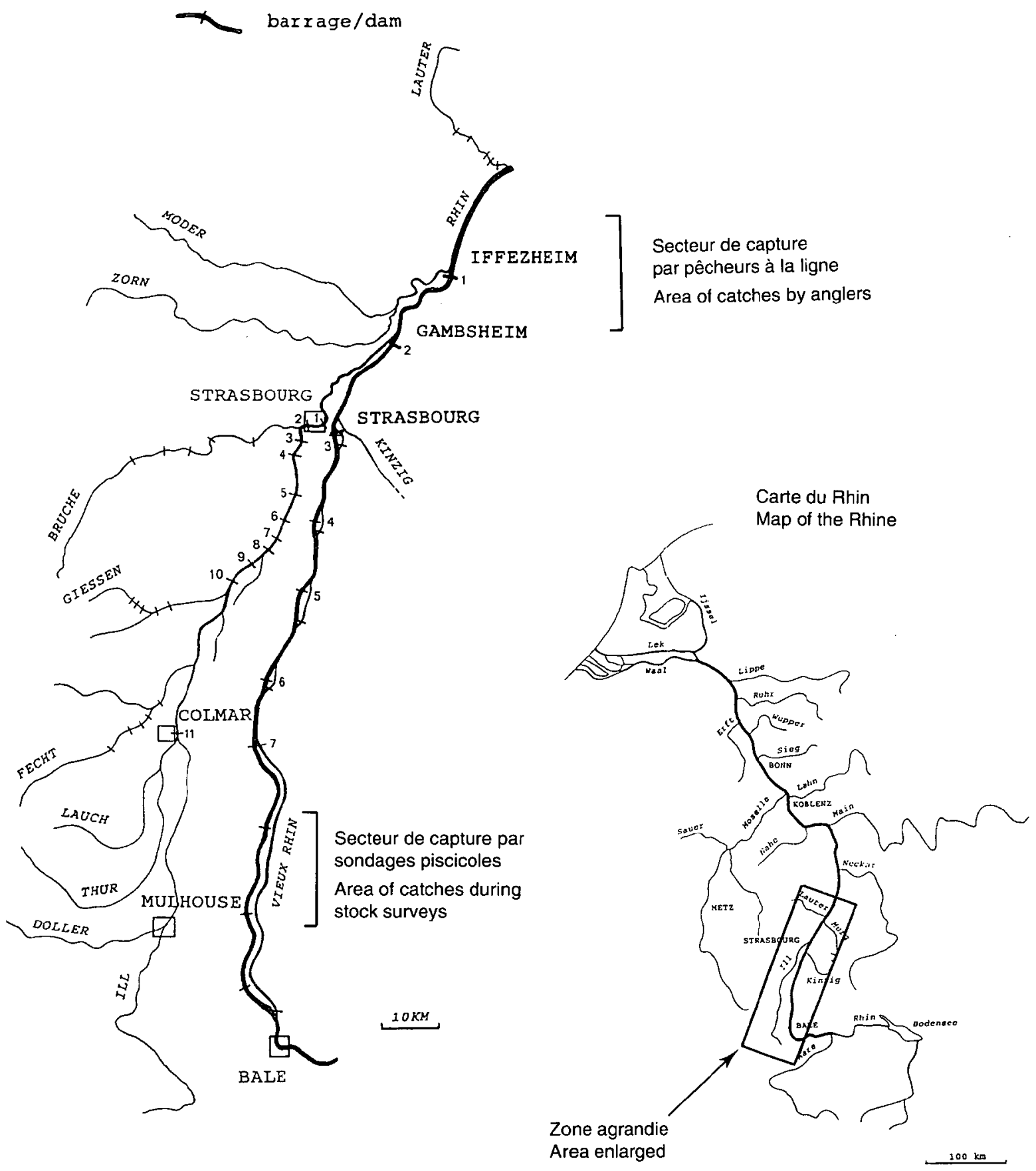

Figure 1 : Carte du secteur d'étude.

Figure 1 : Map of the study area.

- La durée du séjour en rivière est généralement de 1 à 2 ans. La durée du séjour marin est de 1 à 3 hivers, avec une majorité de 2 hivers dans l'échantillon (Tableau I et Fig. 3). Cinq truites du type "mer" ont déjà frayé une fois et un individu semble avoir frayé deux fois.

- Aucune corrélation linéaire n'a pu être établie entre la teneur en strontium et l'âge de mer, la longueur du poisson ou la date de capture (les coefficients de corrélation $\mathrm{R}$ sont respectivement de 0,$37 ; 0,42 ; 0,03$ ). 
Bull. Fr. Pêche Piscic. (1992) 324 : 36-44 - $39-$

Tableau I : Caractéristiques des truites analysées : type mer.

Table I : Characteristics of the trout analyzed : sea type.

\begin{tabular}{|c|c|c|c|c|c|}
\hline date capture & lieu capture $\left(^{*}\right)$ & $\begin{array}{l}\text { longueur totale } \\
(\mathrm{mm})\end{array}$ & $\begin{array}{c}\text { strontium } \\
(\mu \mathbf{g} / \mathbf{g})\end{array}$ & âge rivière & âge mer \\
\hline 10.08 .89 & $\mathrm{R} / \mathrm{F}$ & 706 & 465 & 1 & $2+$ \\
\hline 24.08 .89 & $\mathrm{R} / \mathrm{IF}$ & 500 & 385 & 2 & $1+$ \\
\hline 27.08 .89 & I/GA & 570 & 445 & 2 & $1+$ \\
\hline 11.09 .89 & I/GA & 680 & 326 & 1 & $2+$ \\
\hline 20.09 .89 & I/GA & 840 & 813 & 2 & $3+2,3$ \\
\hline 28.09 .89 & I/GA & 790 & 784 & 2 & $2 t^{2}$ \\
\hline 29.09 .89 & $\mathrm{R} / \mathrm{GA}$ & 570 & 616 & 1 & $1 t^{\prime}$ \\
\hline 06.10 .89 & I/GA & 600 & 131 & 2 & $1+$ \\
\hline 25.11 .89 & $1 / \mathrm{GA}$ & 575 & 333 & 2 & $1+$ \\
\hline 15.07 .90 & $\mathrm{R} / \mathrm{GA}$ & 635 & 332 & 1 & $2 t^{2}$ \\
\hline 21.07 .90 & $\mathrm{R} / \mathrm{ST}$ & 680 & 394 & 1 & $2+$ \\
\hline 22.07 .90 & $\mathrm{R} / \mathrm{GA}$ & 635 & 290 & 1 & $2+$ \\
\hline 22.07 .90 & $\mathrm{R} / \mathrm{GA}$ & 610 & 307 & 2 & $1+$ \\
\hline 24.07 .90 & $\mathrm{R} / \mathrm{GA}$ & 690 & 492 & 2 & $2 t^{2}$ \\
\hline 29.07 .90 & $\mathrm{R} / \mathrm{GA}$ & 800 & 343 & 1 & $3+$ \\
\hline 09.08 .90 & $R / G A$ & 700 & 363 & 1 & $2+$ \\
\hline 09.09 .90 & I/GA & 590 & 291 & 1 & $2 t^{2}$ \\
\hline 12.09 .90 & $\mathrm{R} / \mathrm{IF}$ & 630 & 276 & 1 & $2+$ \\
\hline 28.09 .90 & $\mathrm{R} / \mathrm{GA}$ & 470 & 376 & 2 & $1+$ \\
\hline 06.10 .90 & $\mathrm{R} / \mathrm{GA}$ & 660 & 342 & 1 & $2+$ \\
\hline 08.10 .90 & $\mathrm{R} / \mathrm{GA}$ & 710 & 262 & 1 & $2+$ \\
\hline 28.10 .90 & $\mathrm{R} / \mathrm{GA}$ & 700 & 279 & 2 & $2+$ \\
\hline \multirow{3}{*}{\multicolumn{2}{|c|}{$\begin{array}{l}\text { taille de l'échantillon } \\
\text { moyenne } \\
\text { écart-type }\end{array}$}} & 22 & 22 & & \\
\hline & & 631 & 393 & & \\
\hline & & 155 & 163 & & \\
\hline
\end{tabular}

(*) GA = Gambsheim ; I = Ill ; IF = Iffezheim ; R = Rhin ; ST = Strasbourg.

Tableau II : Caractéristiques des truites analysées : type sédentaire.

Table II : Characteristics of the trout analyzed : sedentary type.

\begin{tabular}{|c|c|c|c|c|}
\hline date capture & lieu capture $\left(^{*}\right)$ & $\begin{array}{l}\text { longueur totale } \\
\qquad(\mathrm{mm})\end{array}$ & $\begin{array}{l}\text { strontium } \\
(\mu \mathrm{g} / \mathrm{g})\end{array}$ & âge \\
\hline 25.08 .89 & I/GA & 470 & 125 & $3+$ \\
\hline 06.10 .89 & I/GA & 440 & 185 & $4+$ \\
\hline 21.06 .90 & $\mathrm{R} / \mathrm{ST}$ & 450 & 214 & $3+$ \\
\hline 09.09 .90 & I/GA & 350 & 235 & $3+^{3}$ \\
\hline \multicolumn{2}{|c|}{ taille du sous-échantillon } & 4 & 4 & \\
\hline \multirow{2}{*}{\multicolumn{2}{|c|}{$\begin{array}{l}\text { moyenne } \\
\text { écart-type }\end{array}$}} & 427 & 190 & \\
\hline & & 53 & 48 & \\
\hline 11.10 .89 & $\mathrm{R} / \mathrm{FE}$ & 364 & 190 & $2+$ \\
\hline 06.11 .89 & $\mathrm{R} / \mathrm{KE}$ & 367 & 254 & $2+$ \\
\hline 06.11 .89 & $\mathrm{R} / \mathrm{KE}$ & 280 & 201 & $2+$ \\
\hline \multirow{3}{*}{\multicolumn{2}{|c|}{$\begin{array}{l}\text { taille du sous-échantillon } \\
\text { moyenne } \\
\text { écart-type }\end{array}$}} & 3 & 3 & \\
\hline & & 337 & 215 & \\
\hline & & 49 & 34 & \\
\hline \multirow{3}{*}{\multicolumn{2}{|c|}{$\begin{array}{l}\text { taille de l'échantillon } \\
\text { moyenne } \\
\text { écart-type }\end{array}$}} & 7 & 7 & \\
\hline & & 389 & 201 & \\
\hline & & 68 & 41 & \\
\hline
\end{tabular}

(*) $\mathrm{FE}=$ Fessenheim ; GA = Gambsheim ; I = Ill ; KE = Kembs ; R = Rhin ; ST = Strasbourg 


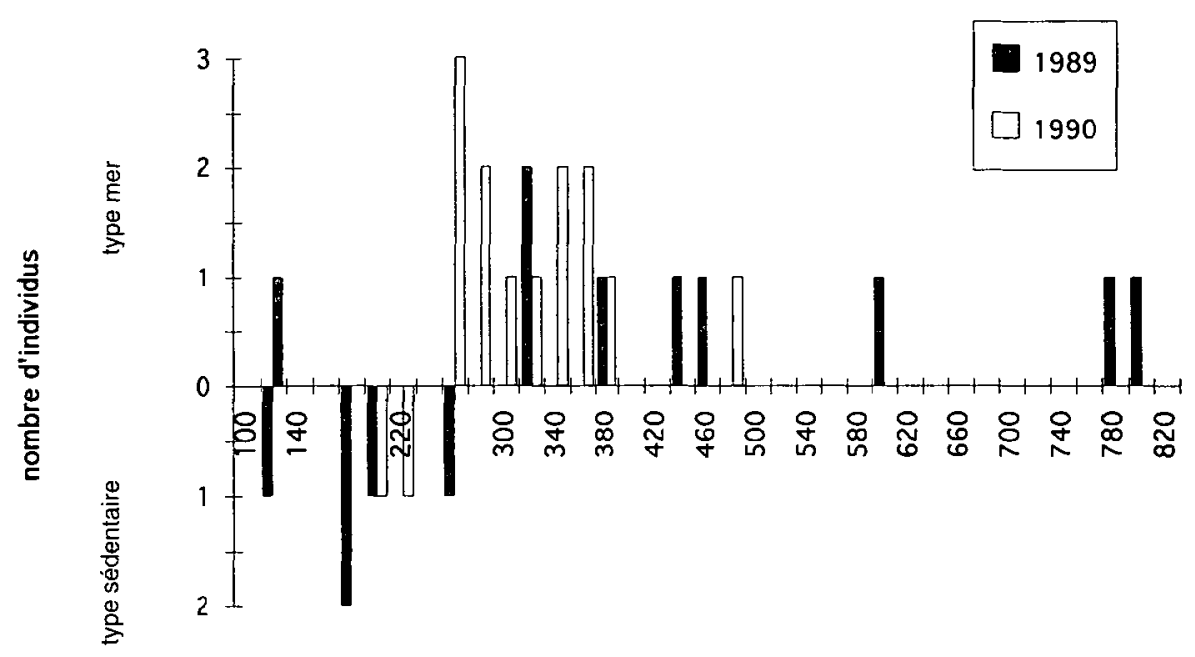

teneur en strontium $(\mu \mathrm{g} / \mathrm{g})$

Figure 2 : Histogramme des teneurs en strontium des écailles analysées.

Figure 2 : Graph of strontium levels found in the scales analyzed.

\section{DISCUSSION - CONCLUSION}

Le Tableau III compare les valeurs de strontium de la présente étude à celles observées par d'autres auteurs pour la même espèce. Les valeurs sont relativement homogènes chez les truites sédentaires, mais varient beaucoup plus largement chez les truites de mer. Les paramètres pouvant influer sur le taux de strontium des écailles sont les suivants :

\section{- Le réseau hydrographique :}

L'influence du réseau hydrographique a été mis en évidence par MOREAU et al. (1983) chez l'omble de fontaine (Salvelinus fontinalis), par GAUSEN et BERG (1988) chez les smolts de saumon atlantique, et par MAISSE et BAGLINIĖRE (1990) chez la truite de mer.

On constate en premier lieu des différences en eau douce, les truites de l'échantillon de BAGENAL et al. (1973) ayant des valeurs nettement plus faibles que celles des échantillons du Nord-Ouest de la France et du Rhin. Les différences constatées sont probablement liées à la teneur en strontium des rivières d'origine. A titre indicatif, RILEY et CHESTER (1971) donnent une valeur moyenne de $50 \mu \mathrm{g} / \mathrm{l}$ pour l'eau douce, tandis que la teneur en strontium du Rhin était beaucoup plus élevée dans les années 1960-1980, au maximum de la pollution de ce fleuve (330 à $3300 \mu \mathrm{g} / \mathrm{l}$ selon SALIOU, 1990), qu'elle ne l'est aujourdh'ui $(<300 \mu \mathrm{g} / \mathrm{l})$. Ces teneurs pourraient expliquer les valeurs étonnantes observées chez les truites de mer du Rhin Moyen en 1981-1982 par OLBRICH (1984), qui ne les compare malheureusement pas à des valeurs-témoins de truites sédentaires.

En deuxième lieu, les écarts observés chez les truites de mer du Rhin sont très importants comparativement à ceux des autres échantillons du Tableau III, certaines valeurs étant très proches de valeurs observées chez les truites sédentaires. Ces écarts sont probablement le résultat des particularités de l'estuaire, aménagé pour lutter contre la pénétration d'eau salée grâce à des digues équipées de clapets : les truites dévalant le Rhin peuvent emprunter soit l'exutoire principal qui conduit directement à la mer, soit l'un des exutoires secondaires qui aboutissent dans des grands lacs estuariens peu salés et d'une grande richesse biologique (Kettelmeer, ljsselmeer, Haringvliet). La présence de truites de mer dans les deux types de milieux a été montrée à l'occasion d'une expérience de marquage en Hollande, qui a également permis de mettre en évidence la possibilité de migration d'adultes depuis chacun de ces milieux 
jusqu'au Rhin Supérieur. On se trouverait donc dans le Rhin en présence de deux types de truites dont la structure des écailles est du type "mer" : des truites de mer à proprement parler, et des truites de lac estuarien, dont les teneurs en strontium sont intermédiaires entre celles des truites d'eau douce et celles des truites de mer (valeurs de 250 à $400 \mu \mathrm{g} / \mathrm{l}$ de la figure 2).

\section{Tableau III : Comparaison des teneurs en strontium d'écailles de truites de différentes origines.}

Table III : Comparison of strontium levels in scales of trout of different origins.

\begin{tabular}{|c|c|c|c|c|c|c|c|}
\hline auteurs & origine des & ruites (S. trutta) & nombre & $\operatorname{mini}$ & $\operatorname{maxi}$ & moyenne & écart-type \\
\hline \multirow[t]{2}{*}{ Présente étude } & Rhin Supérieur & type sédentaire & 7 & 125 & 254 & 201 & 41 \\
\hline & & type mer & 22 & 131 & 813 & 393 & 163 \\
\hline OLBRICH, 1984 & Rhin Moyen/Aval & type mer & 4 & 699 & 1452 & 1002 & 363 \\
\hline \multirow{2}{*}{$\begin{array}{l}\text { BAGENAL et al., } \\
1973\end{array}$} & Angleterre & rivière & 13 & 52 & 142 & 102 & 26 \\
\hline & & mer & 16 & 320 & 683 & 412 & 100 \\
\hline MAISSE et & rivière Oir & type sédentaire & 18 & & & 247 & 53 \\
\hline \multirow[t]{3}{*}{ BAGLINIĖRE, 1990} & & type mer (automne) & 15 & & & 220 & 34 \\
\hline & rivière Calonne & type mer (automne) & 20 & & & 155 & 54 \\
\hline & & type mer (été) & 19 & & & 265 & 74 \\
\hline \multirow[t]{4}{*}{ SALIOU, 1991} & Bretagne & élevage eau douce & 9 & 195 & 231 & 215 & 13 \\
\hline & & élevage mer 13 mois & 8 & 472 & 674 & 529 & 66 \\
\hline & & élevage mer 15 mois & 20 & 521 & 687 & 600 & 55 \\
\hline & & élevage mer 19 mois & 10 & 438 & 650 & 530 & 67 \\
\hline
\end{tabular}

\section{- Le temps de séjour en mer et la date de capture}

L'importance de ces paramètres a été mise en évidence par MAISSE et BAGLINIĖRE (1990) pour les truites de l'Oir. Ces auteurs montrent que les teneurs en strontium sont trop faibles chez les truites restées moins d'un an en mer pour pouvoir les distinguer des truites de rivière. De plus, ils observent une baisse des teneurs en strontium à l'automne, ce qui nuit à cette méthode de distinction. Cette baisse pourrait être due à une mobilisation du strontium des écailles avec la maturation sexuelle, le temps de séjour en rivière n'étant pas en cause. Dans le cas du Rhin, aucune relation de ce type n'apparaît à la lueur de l'échantillon étudié dans lequel un seul poisson a été capturé après le 1er novembre. Par ailleurs, il faut noter les valeurs élevées de l'échantillon de OLBRICH pour des truites capturées en hiver.

En conclusion, les résultats des analyses effectuées du Rhin sur de grandes truites montrent que l'identification du type truite de mer à partir des écailles peut être une méthode performante, malgré l'existence probable dans ce bassin d'une sous-population d'estuaire moins marquée par le strontium.

Une première caractérisation des truites de mer du Rhin est possible à partir de l'échantillon récolté : il s'agit en majorité de poissons à long séjour marin, comme sur l'Orne, rivière de Normandie (RICHARD et BAGLINIĖRE, 1990), mais la proportion d'individus ayant séjourné un an en rivière est plus faible dans le Rhin (EUZENAT et al., 1991). La taille moyenne des poissons de l'échantillon étudié $(63 \mathrm{~cm})$ est voisine de celle d'autres échantillons déjà récoltés dans le Rhin : $69 \mathrm{~cm}(\mathrm{n}=8, \sigma=14)$ pour l'échantillon de OLBRICH (1984); $61 \mathrm{~cm}(n=20, \sigma=7)$ pour l'échantillon de WEIBEL (1991).

L'origine des truites anadromes remontant le Rhin Supérieur reste mal connue, les frayères accessibles aux adultes dans cette partie du cours d'eau ou dans ses affluents étant limitées à quelques sites, souvent de qualité médiocre (ROCHE, 1991). La dévalaison de truites juvéniles en provenance de l'amont du Rhin Supérieur ou de l'Ill est régulièrement 
constatée au printemps, notamment à l'amont des centrales hydroélectriques où elles se concentrent. L'observation des nageoires et des écailles montre que certains de ces poissons sont issus de repeuplement. La dévalaison de truites de rivière a donc lieu dans le Rhin, phénomène similaire à celui décrit par ARRIGNON (1968) dans le bassin de la Seine. La dévalaison de juvéniles de truites de mer élevées en pisciculture a par ailleurs été montrée par la recapture en estuaire de poissons marqués dans la Sieg, affluent du Rhin Moyen (STEINBERG et LUBIENIECKI, 1991).

Figure 3 : Exemples d'écailles de grandes truites du Rhin. Position des bandes hivernales.

Figure 3 : Examples of scales of large trout from the Rhine. Position of the winter bands.

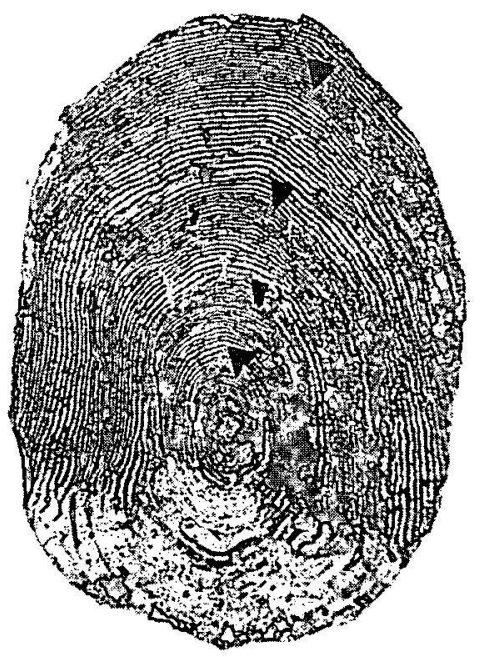

Figure 3a : Type truite de mer, Rhin Gambsheim, 28.10.90, $700 \mathrm{~mm}, 2.2+$

Figure 3a : Sea trout type, Rhine Gambsheim, 28.10.90, $700 \mathrm{~mm}, 2.2+$

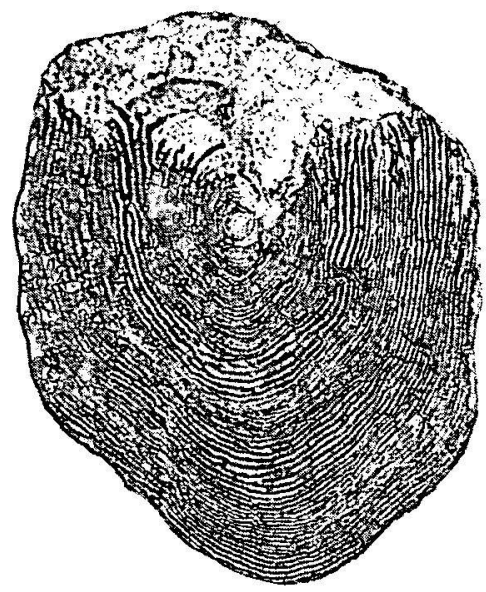

Figure 3c : Type truite de mer, Rhin Gambsheim, 28.09.90, $470 \mathrm{~mm}$, interprétation difficile, $2.1+$ probable

Figure 3c : Sea trout type, Rhine Gambsheim, 28.09.90, $470 \mathrm{~mm}$, difficult to read, probably $2.1+$

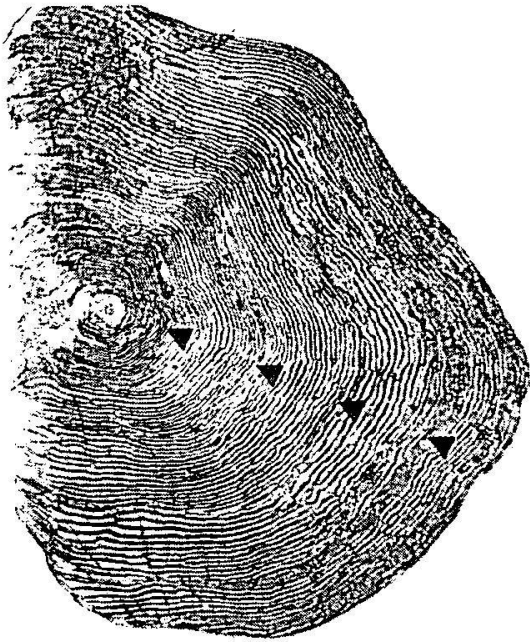

Figure 3b : Type truite de mer, Rhin Gambsheim, 24.07.90, $800 \mathrm{~mm}, 1.3+$

Figure 3b : Sea trout type, Rhine Gambsheim, 24.07.90, $800 \mathrm{~mm}, 1.3+$

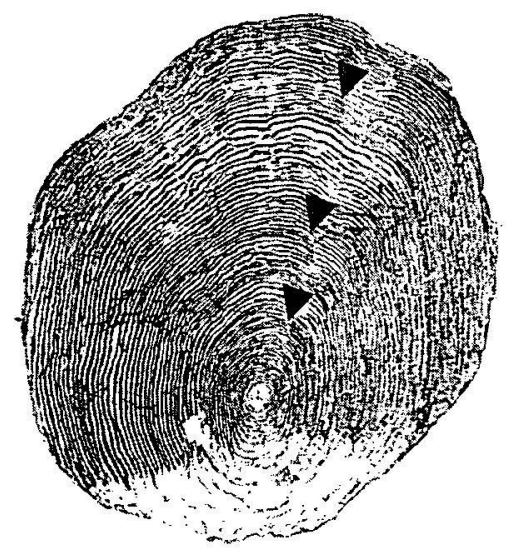

Figure 3d : Type truite sédentaire, III Gambsheim, 25.08.89, $470 \mathrm{~mm}, 3+$

Figure 3d : Sedentary trout type, Ill Gambsheim, $25.08 .89,470 \mathrm{~mm}, 3+$ 
La truite de mer était autrefois présente dans le bassin du Rhin mais ses effectifs étaient faibles par rapport à ceux du saumon (GROOT, 1989 ; STEINBERG et LUBIENIECKI, 1991). Selon les estimations de captures données par GROOT (1989) pour la Hollande entre 1886 et 1986, la truite de mer n'aurait pas connu le déclin observé chez le saumon et serait toujours restée présente en Hollande. II semble que la truite de mer a de nouveau colonisé la partie moyenne et supérieure du Rhin lorsque les conditions de migration sont redevenues correctes. Sa réapparition en amont de l'estuaire correspond de fait à une amélioration de la qualité de l'eau, notamment une élévation du taux d'oxygène dissous (CAZEMIER, 1988). D'autres espèces anadromes ont plus récemment fait leur apparition dans le Rhin Moyen et Supérieur : la lamproie fluviatile (Lampetra fluviatilis), la lamproie marine (Petromyson marinus) et le saumon atlantique (Salmo salar) (WEIBEL, 1991 ; STEINBERG et al., 1991). La mise en évidence d'individus isolés ne signifie pas pour autant que ces populations soient rétablies puisqu'elles sont, en totalité pour les saumons et en partie pour les truites de mer, tributaires d'un soutien artificiel. Seul le rétablissement du cycle biologique complet, parl'accès aux zones de reproduction pour un nombre suffisant d'individus, sera le signe d'une réinstallation durable de ces espèces.

\section{REMERCIEMENTS}

Cette étude a été possible grâce à la participation de quelques pêcheurs, notamment R. et G. ROEHM, qui ont assuré le relais entre le CSP et les autres pêcheurs. Les analyses de strontium ont été financées dans le cadre de la convention entre le CSP et le Ministère de l'Environnement-DRAE Alsace (restauration des écosystèmes rhénans - objectif saumon). Enfin, nous remercions J.L. BAGLINIĖRE de l'INRA de Rennes pour son aide dans l'interprétation scalimétrique et la rédaction de ce document.

\section{BIBLIOGRAPHIE}

ARRIGNON J., 1968. Comportement de l'espèce Salmo trutta dans le bassin de la Seine. Bull. Fr. Piscic., 229, 117-122.

BAGENAL T.B., MACKERETH F.J.H., HERON J., 1973. The distinction between brown trout and sea trout by the strontium content of their scales. J. Fish. Biol., 5, 555-557.

BAGLINIÈRE J.L., 1991. La truite commune (Salmo trutta L.), son origine, son aire de répartition, ses intérêts économique et scientifique. In "La truite, biologie et écologie", J.L. Baglinière et G. Maisse ed., INRA Paris, 11-22.

CAZEMIER W.G., 1988. Fish and their environment in large European river ecosystems. The Dutch part of the River Rhine. Rev. Sciences de l'Eau, 7 (1), 95-114.

CAZEMIER W.G., 1991. The migration of sea trout (Salmo trutta trutta L. ) along the Dutch coast and in the lower part of the Rhine. RIVO-DLO, ljmuiden, 27 p. + tab.

EUZENAT G., FOURNEL F., RICHARD A., 1991. La truite de mer en Normandie/Picardie. In "La truite, biologie et écologie", J.L. Baglinière et G. Maisse ed., INRA Paris, 183-213.

GAUSEND., BERG O.K., 1988. Strontium levels in scales and vertebrae of wild atlantic salmon, Salmo salar L., smolts. Prog. Fish-Cult., 49, 34-39.

GROOT (de) S.J., 1989. Literature survey into the possibility of restocking the River Rhine and its tributaries with sea trout (Salmo trutta trutta). RIVO, ljmuiden, Publication and reports Vol. 1989-12 of the project "Ecological Rehabilitation of the River Rhine", $53 \mathrm{p}$.

MAISSE G., BAGLINIĖRE J.L., 1990. Limites de la méthode de reconnaissance de l'origine (marine ou dulcicole) de la truite (Salmo trutta L.) au moyen de l'analyse de la teneur en strontium des écailles. Bull Fr. Pêche Piscic., 316, 24-27.

MOREAU G., BARBEAU C., FRENETTE J.J., SAINT-ONGE J., SIMONEAU M., 1983. Zinc, manganese and strontium in opercula and scales of brook trout (Salvelinus fontinalis) as indicators of lake acidification. Can. J. Fish. Aquat. Sci., 40, 1685-1691. 
OLBRICH P., 1984. Untersuchungen zum Wiedererscheinen der Meerforelie (Salmo trutta L.) im oberen Niederrheingebiet. Der Fischwirt, 33 (3), 22-24.

PHILIPPART J.C., 1983. Note sur la redécouverte de "truites de mer" dans un affluent de la Meuse liégeoise en 1983. Cahiers d'Ethologie appliquée, 3(1), 105-114.

RICHARD A., BAGLINIĖRE J.L., 1990. Description et interprétation des écailles de truites de mer (Salmo trutta L.) de deux rivières de Basse-Normandie : I'Orne et la Touques. Bull. Fr. Pêche Piscic., 319, 239-257.

RILEY J.P., CHESTER R., 1971. Introduction to marine chemistry. Academic Press, London.

ROCHE P., 1991. Le saumon du Rhin : habitats et stocks potentiels en partie française. Rapport CSP Metz - Min. Environnement - DRAE Alsace-Sandoz, $36 \mathrm{p}$.

SALIOU P., 1990. Distinction entre truite de mer et truite d'eau douce par l'étude de la concentration en strontium des écailles. Thèse Ecole Nationale Vétérinaire Nantes, 108 p.

STEINBERG L. LUBIENIECKI B., 1991. Die Renaissance der Meer-forelle (Salmo trutta trutta L.) und erste Versuche zur Wiedereinbürgerung des Lachses (Salmo salar L.) in Nordrhein-Westfalen. Fischökologie, 5, 19-33.

STEINBERG L., MARMULLA G., SCHMIDT G.,LEMMANN J. 1991. Erstergesicherter Nachweiss des Lachses (Salmo salarL.) im Gewässersystem der Sieg seilt über drei Jahrzehnten. Fischökologie Aktuell, Heft 5, 2-3.

WEIBEL U. (1991). Neue Ergebnisse zur Fischfauna des nördlichen Oberrheinsermittelt im Rechengut von Kraftwerken. Fischökologie, 5, 43-68. 